\title{
March 2016 Imaging Case of the Month
}

\author{
Michael B. Gotway, MD \\ Department of Radiology \\ Mayo Clinic Arizona \\ Scottsdale, AZ USA
}

Clinical History: A 66 year-old man with orthotopic heart transplantation 1 year previously presented with complaints of recent-onset small volume ( $<1$ teaspoon) hemoptysis, post-nasal drip, and night sweats. The patient indicated he had recent contact with several young grandchildren who had upper respiratory tract symptoms. The patient's past medical history was remarkable for recurrent constrictive pericarditis (surgically treated), hypertension, type II diabetes mellitus (treated with insulin), psoriasis, sleep-disordered breathing, and grade 2 cardiac transplant rejection diagnosed 6 months earlier. The patient's medication list included insulin, Cellcept (mycophenolate mofetil), Prograf (tacrolimus), prednisone, among others. On physical examination, the patient was mildly tachycardic (heart rate $=104$ beats $/$ minute) with an oxygen saturation on room air of $92 \%$. The white blood cell count was within the normal range, but C-reactive protein and B-type natriuretic peptide levels were reportedly elevated.

Frontal chest radiography (Figure 1) was performed, with a radiograph from one month other also shown for comparison.

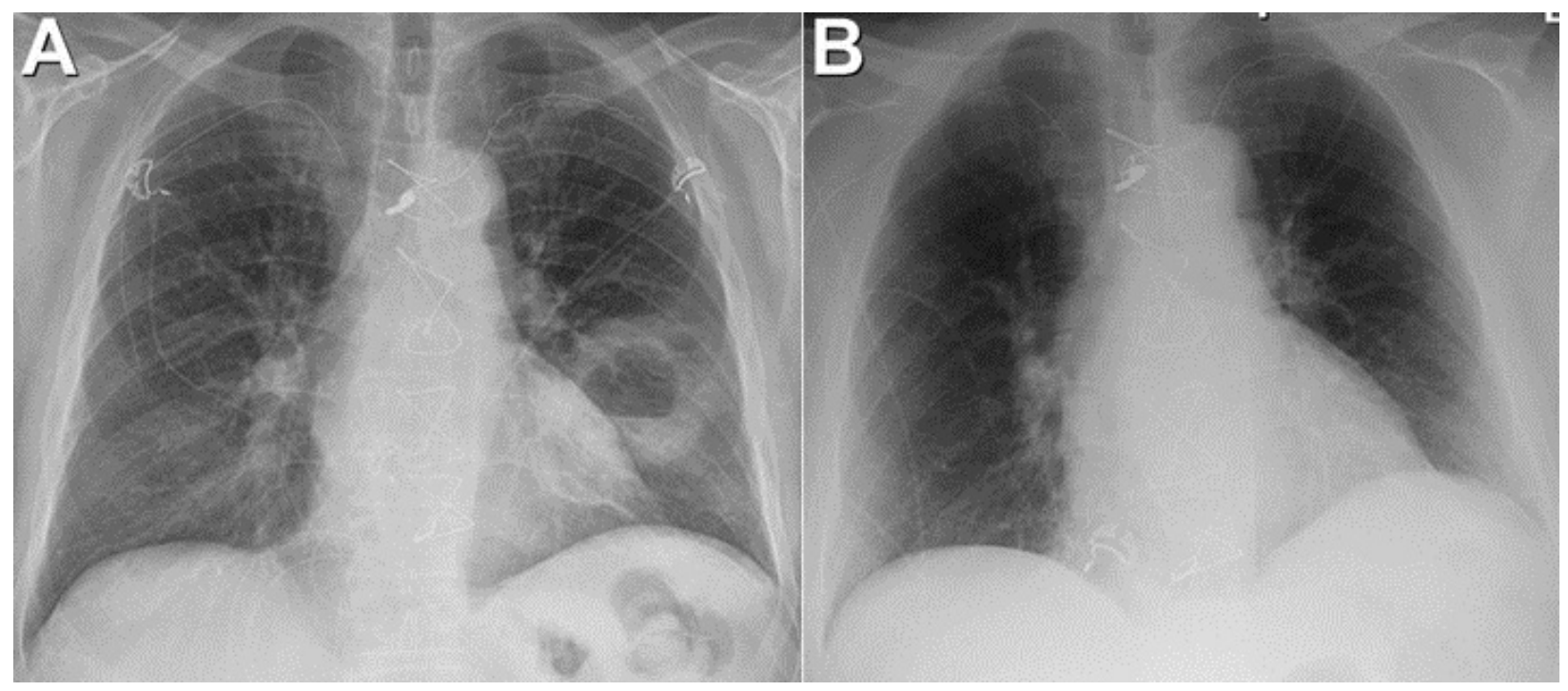

Figure 1. Frontal $(A)$ chest radiography shows interval development of a thick-walled left lower lobe cavity since a chest radiograph performed one month previously. 
Which of the following statements regarding the chest radiograph is most accurate?

1. The frontal chest radiograph shows a new lung cavity

2. The frontal chest radiograph shows basal predominant fibrotic abnormalities

3. The frontal chest radiograph shows large lung volumes with a cystic appearance

4. The frontal chest radiograph shows new peribronchial lymph node enlargement

5. The frontal chest radiograph shows new poorly defined nodular opacities bilaterally 


\section{Correct!}

\section{The frontal chest radiograph shows a new lung cavity}

The presentation radiograph shows a moderately thick-walled left lower lobe cavity with poorly defined margins, entirely new since the comparison chest radiograph one month previously. No other lung nodules are evident, and no clear evidence of peribronchial or mediastinal lymph node enlargement is seen. Lung volumes appear normal, with no features to suggest fibrotic lung disease, and no evidence of cystic lung disease is seen.

The patient underwent enhanced thoracic CT (Figure 2) to further characterize the lesion found at chest radiography.

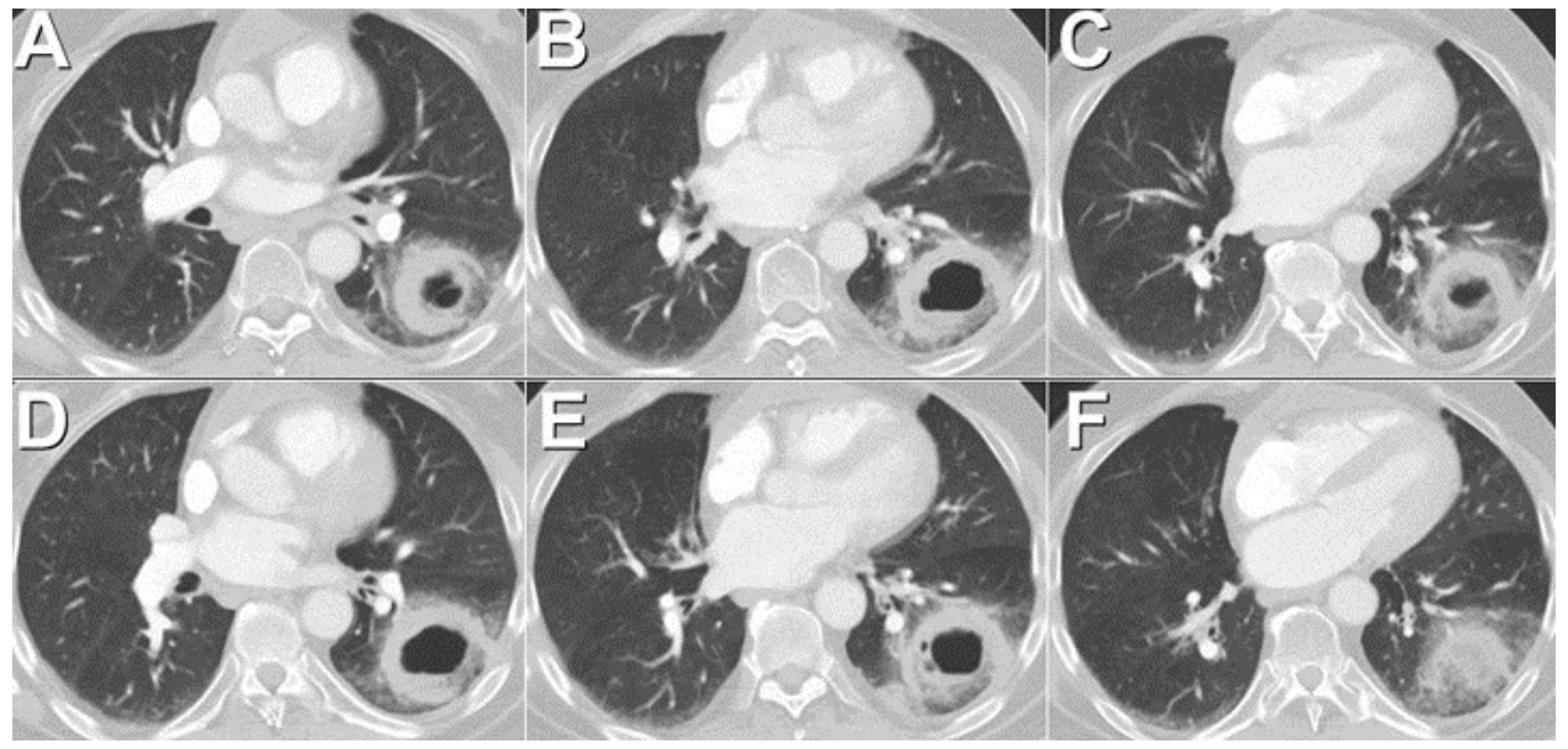

Figure 2. Axial enhanced thoracic CT displayed in lung windows.

Which of the following is correct regarding the description of the thoracic CT findings?

1. Thoracic CT confirms the chest radiographic findings and reveals additional lung nodules

2. Thoracic CT confirms the chest radiographic findings and shows no other relevant findings

3. Thoracic CT shows the left lower lobe lesion demonstrate the "waterlily" sign

4. Thoracic CT shows the left lower lobe lesion demonstrates an "air crescent" sign

5. Thoracic CT shows the left lower lobe lesion demonstrates the "reverse groundglass halo" ("atoll") sign 


\section{Correct! \\ 2. Thoracic CT confirms the chest radiographic findings and shows no other relevant findings}

Thoracic CT confirms the presence of a relatively thick-walled cavitary lesion in the left lower lobe and shows that the lesion is surrounded by ground-glass opacity; the latter likely reflects hemorrhage. No other lung nodules are present. The "air crescent" sign is not present- this sign appears as a thin rim or air or gas density around a nodule or mass contained within a cavity and is commonly seen with mycetomas or in the context of invasive fungal infection when immune system recovery occurs. The "reverse groundglass halo ("atoll") sign appears as a rounded area of ground-glass attenuation surrounded by consolidation, manifesting either as a complete or nearly complete ring; this sign is commonly seen with organizing pneumonia, but may be seen with invasive fungal infections, pulmonary embolism with hemorrhage and infarction, granulomatous infection, and sarcoidosis, among other etiologies. In this case, the internal aspect of the lesion is mostly gas, not ground-glass opacity, so the "reverse ground-glass halo" sign is not present. The "waterlily" sign is present when a cavity contains an internal opacity with a wavy contour that appears to "float" on an air-fluid level within the cavity, and is typically seen in the context of echinococcosis.

At this point, which of the following differential diagnostic considerations is not appropriate for the imaging findings in this patient?

1. Bacterial abscess

2. Fungal pneumonia

3. Mixed anaerobic pleuropulmonary pneumonia

4. Nocardiosis

5. Pneumocystis jirovecii pneumonia 


\section{Correct! \\ 5. Pneumocystis jirovecii pneumonia}

Pneumocystis jirovecii pneumonia commonly presents as multifocal, bilateral groundglass opacity; focal cavitary lung disease would be an extremely unusual presentation for this infection. The other choices commonly present with focal cavitary lung disease and certainly could affect an immunocompromised transplant recipient.

The patient underwent sputum induction which revealed both rhinoviral and enteroviral RNA. The patient's immunosuppression regimen was decreased and additional antifungal therapy with voriconazole was presumptively begun.

Which of the following represents an appropriate step for the evaluation of this patient?
1. ${ }^{18}$ FDG-PET scanning
2. Bronchoscopy
3. Extended cervical mediastinoscopy
4. Percutaneous transthoracic needle biopsy
5. Video - assisted thoracoscopic surgical biopsy 


\section{Correct! \\ 2. Bronchoscopy}

Bronchoscopy is the best choice for the evaluation of this patient. Percutaneous transthoracic needle biopsy could be considered, but biopsy of cavitary lesions can be problematic and result in complications. Video - assisted thoracoscopic surgical biopsy would certainly be capable of establishing the etiology of the cavity, but is needlessly invasive and may be difficult, short of an actual lobectomy, given the size of the lesion. Extended cervical mediastinoscopy is occasionally employed to sample left mediastinal lymph nodes, most commonly in the context of staging primary malignancy in the left lung, to establish or exclude N2 I mediastinal disease; however, this patient has no leftsided lesion to target with this procedure. ${ }^{18} \mathrm{FDG}$-PET scan would provide little useful information in this patient- lack of tracer utilization would not allow the lesion to be managed conservatively, and elevated tracer uptake within the lesion would be nonspecific and not distinguish among the myriad of infectious and non-infectious diagnostic considerations for this lesion.

The patient underwent bronchoscopy with bronchial lavage directed into the left lower lobe. At bronchoscopy, purulent secretions were noted emanating from the superior segment left lower lobe bronchus. Numerous white blood cells were noted in the bronchial lavage fluid. Testing of the bronchial lavage fluid showed normal respiratory flora, moderate gram + cocci and bacilli, few gram - bacilli and Moraxella catarrhalis. Polymerase chain reaction testing for Pneumocystis jirovecii, Epstein-Barr virus, coccidioidomycosis, Legionella, cytomegalovirus, and Mycobacterium tuberculosis were negative. No evidence of Nocardia spp. was noted on smear. Aspergillus antigen (0.876) was detected. The patient was placed on broad-spectrum antibiotics and additional antifungal therapy was instituted. His symptoms continued to worsen, with increasing shortness of breath and recurrent hemoptysis noted. Repeat thoracic CT (Figure 3) was performed.

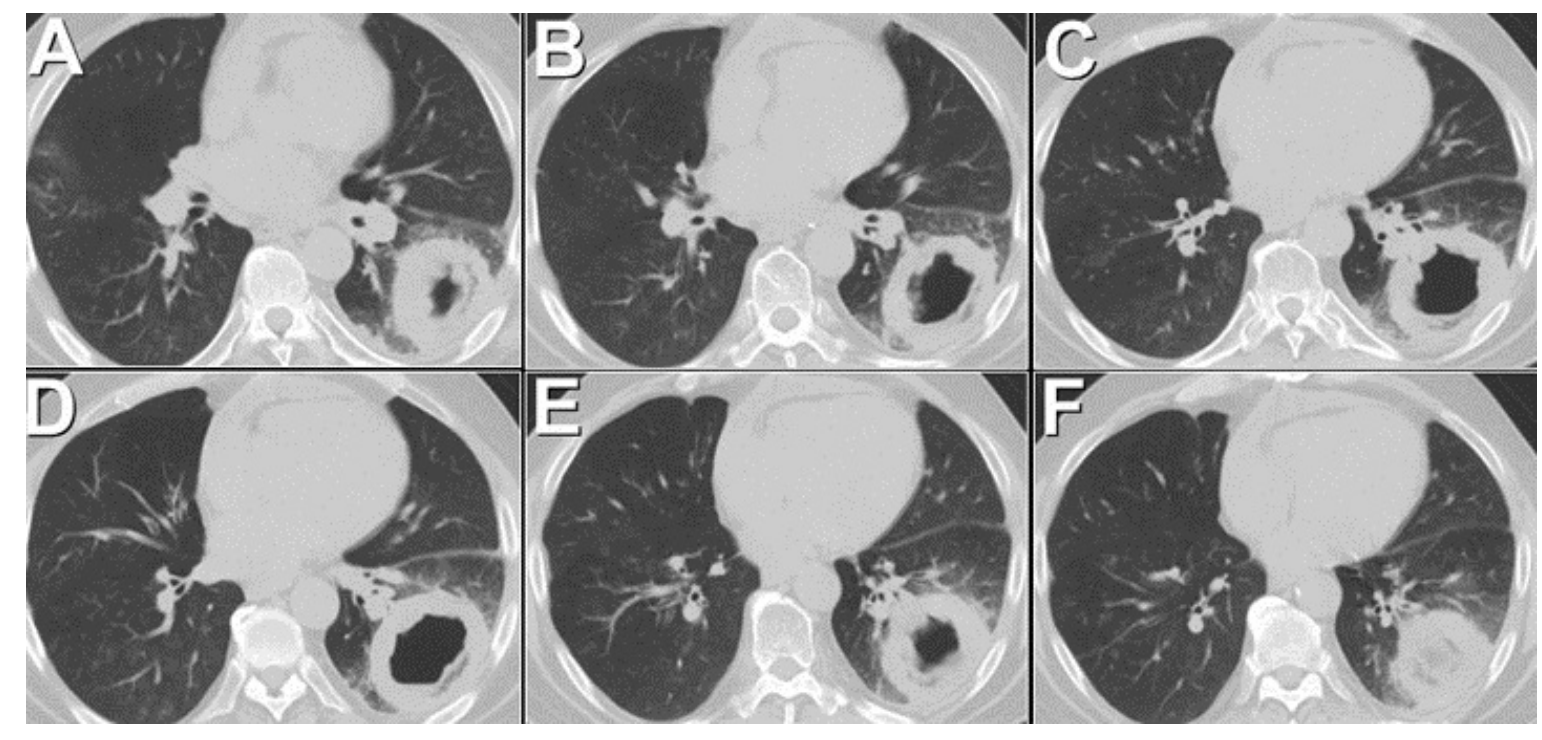

Figure 3. Repeat axial enhanced thoracic CT displayed in lung windows. 
Regarding this examination, which of the following is correct?

1. The repeat thoracic CT shows development of an opacity within the left lower lobe cavity suggesting mycetoma

2. The repeat thoracic CT shows interval decrease in size of the left lower lobe cavity

3. The repeat thoracic CT shows interval development of numerous small solid nodules suggesting a superimposed hematogenous process

4. The repeat thoracic CT shows interval enlargement of the left lower lobe cavity

5. The repeat thoracic CT shows left-sided hydropneumothorax suggesting development of bronchopleural fistula 


\section{Correct! \\ 4. The repeat thoracic CT shows interval enlargement of the left lower lobe cavity}

There is no pleural abnormality and, while some patchy areas of faint ground-glass opacity are present, particularly in the right upper and lower lobes (possibly reflecting areas of aspirated blood given the hemoptysis) no evidence of new randomly distributed bilateral small solid nodules to suggest development of a hematogenous infectious process is seen.). The left lower lobe cavity has enlarged since the CT performed one week earlier (Figure 2). However, there is no evidence of solid internal opacity within the cavity to indicate development of mycetoma.

Based on the data thus far, which of the following represents the next most appropriate step for the evaluation of this patient?

1. Broaden antimicrobial coverage for bacterial infection

2. Consider left lower lobe surgical resection

3. Continue current treatment plan and monitor for response

4. Percutaneous transthoracic needle biopsy

5. Repeat flexible fiberoptic bronchoscopy 


\section{Correct! \\ 2. Consider left lower lobe surgical resection}

Thoracic surgery was consulted for possible left lower lobe resection. Given that the patient is already on extensive broad-spectrum anti-bacterial and anti-fungal treatment, yet the cavity has progressed in size and the patient's symptoms continue to worsen and no additional potential infectious etiologic agents have been recovered following extensive investigations, continuing the current course of therapy is ill-advised. The previous bronchoscopic procedure was successful and provided data that made a number of differential diagnostic considerations unlikely; repeating the procedure is therefore unlikely to provide additional, management-altering information.

Thoracoscopic resection of the left lower lobe is certainly feasible, although thoracoscopic biopsy, short of complete left lower lobe resection, would provide difficult given the size of the left lower lobe cavity. Percutaneous transthoracic needle biopsy could be considered but is sometimes avoided with cavity lung lesions due to a perceived higher risk of complications, and the likelihood that percutaneous transthoracic needle biopsy would add additional information to that already obtained through bronchoscopy is low. Left lower lobe resection would be both diagnostic and therapeutic for this patient.

The patient underwent video-assisted thoracoscopic left lower lobe resection. The histopathologic specimen from this procedure is shown in Figure 4.

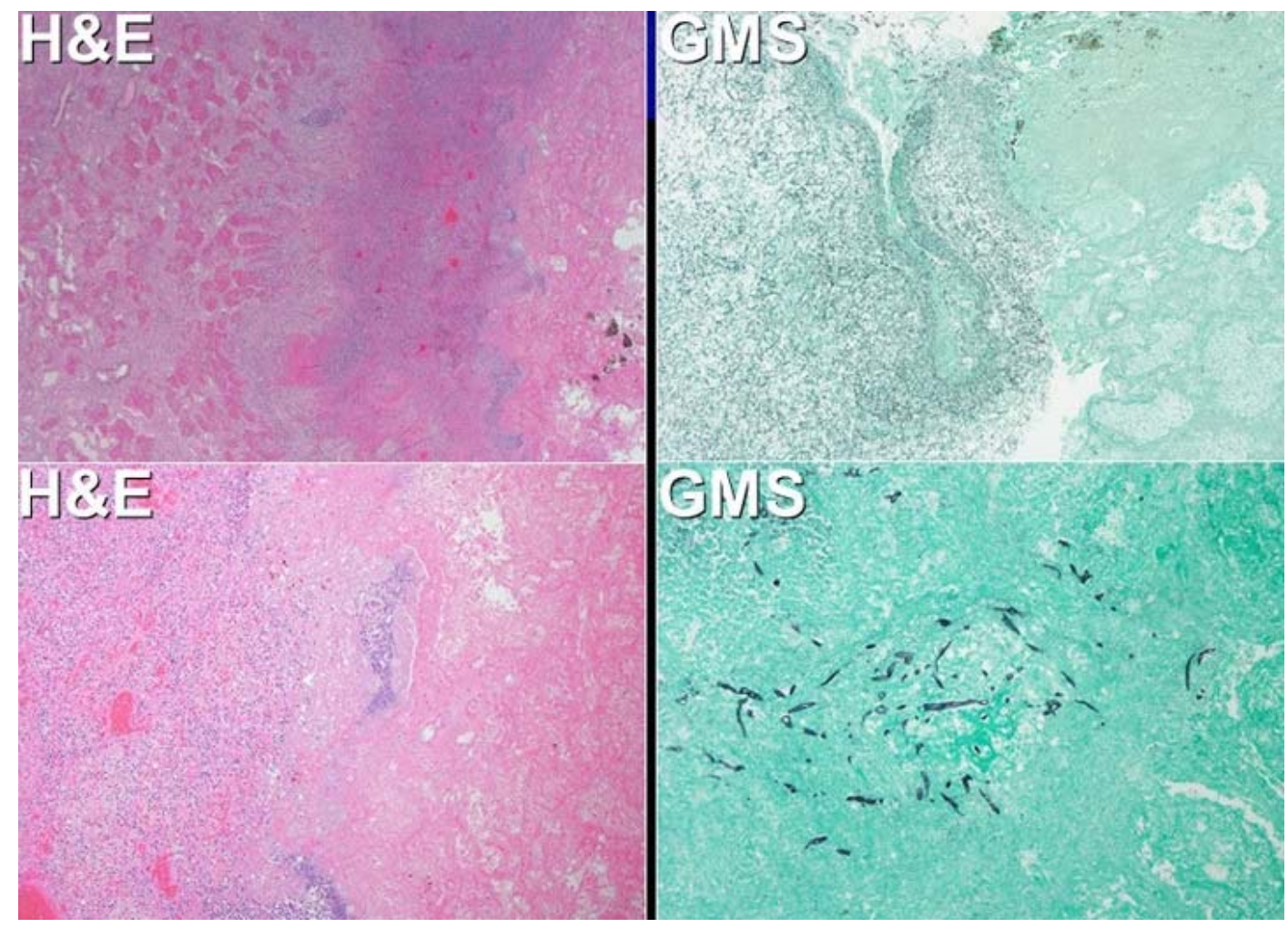

Figure 4. Histopathological specimen: $A$ and $B=$ Hematoxylin and eosin stains. $C$ and $D=$ Grocott-Gomori's (or Gömöri) methenamine silver stain. 
Which of the following statements is most accurate regarding interpretation of the histopathologic specimen?

1. The histopathologic specimen shows coccidioidomycosis spherules

2. The histopathologic specimen shows necrotic lymphoproliferative tumor

3. The histopathologic specimen shows obliterative arteritis

4. The histopathologic specimen shows pauciseptate fungal hyphae suggesting angio-invasive fungal infection

5. The histopathologic specimen shows weakly acid-fast organisms consistent with mycobacterial infection 


\section{Correct! \\ 4. The histopathologic specimen shows pauciseptate fungal hyphae suggesting angio-invasive fungal infection}

The H\&E (hematoxylin and eosin) stains shows areas of fibrinous injury and scattered hemosiderin-laden macrophages. There is no evidence of necrotic lymphoproliferative tumor or obliterative arteritis. In addition small collections of eosinophils and rare neutrophils are identified. Hypervacuolated macrophages are also present. The biopsy is negative is negative for HSV1, HSV2, and cytomegalovirus. The Grocott-Gomori's (or Gömöri) methenamine silver stain and acid-fast bacilli stains shows the cells walls of the organisms that are present to be stained brown-black, but no acid-fast organisms (including weakly acid-fast organisms) are present, nor are the spherules of coccidioidomycosis evident. The morphology of the organisms show a pauciseptate hyphal appearance, suggestive of Mucorales fungi (Rhizopus species, Mucor species, Rhizomucor species, among others, and are consistent with angio-invasive fungal infection. However, other invasive fungi, such as Aspergillus, Fusarium, Scedosporium, and Penicillium species, can appear somewhat similarly on tissue sections and culture is required for proper species identification.

Within a few days, Rhizopus spp. was identified in culture. The patient was started on isovuconazole and improved over the next few weeks.

Diagnosis: Angio-invasive fungal infection due to Rhizopus (zygomycosis / mucormycosis.

\section{References}

1. Chung JH, Godwin JD, Chien JW, Pipavath SJ. Case 160: Pulmonary mucormycosis. Radiology. 2010 Aug;256(2):667-70. [CrossRef] [PubMed]

2. Georgiadou SP, Sipsas NV, Marom EM, Kontoyiannis DP. The diagnostic value of halo and reversed halo signs for invasive mold infections in compromised hosts. Clin Infect Dis. 2011 May;52(9):1144-55. [CrossRef] [PubMed]

3. Marom EM, Kontoyiannis DP. Imaging studies for diagnosing invasive fungal pneumonia in immunocompromised patients. Curr Opin Infect Dis. 2011 Aug;24(4):309-14. [CrossRef] [PubMed]

4. Georgiadou SP, Pongas G, Fitzgerald NE, Lewis RE, Rytting M, Marom EM, Kontoyiannis DP. Invasive mold infections in pediatric cancer patients reflect heterogeneity in etiology, presentation, and outcome: a 10-year, single-institution, retrospective study. J Pediatric Infect Dis Soc. 2012 Jun;1(2):125-35. [CrossRef] [PubMed]

5. Marchiori E, Marom EM, Zanetti G, Hochhegger B, Irion KL, Godoy MC. Reversed halo sign in invasive fungal infections: criteria for differentiation from organizing pneumonia. Chest. 2012 Dec;142(6):1469-73. [CrossRef] [PubMed]

6. Wahba H, Truong MT, Lei X, Kontoyiannis DP, Marom EM. Reversed halo sign in invasive pulmonary fungal infections. Clin Infect Dis. 2008 Jun 1;46(11):1733-7. [CrossRef] [PubMed] 
7. Godoy MC, Marom EM. Reversed halo sign in pulmonary zygomycosis. Thorax. 2011 Jun;66(6):544. [CrossRef] [PubMed]

8. Algın O, Gökalp G, Topal U. Signs in chest imaging. Diagn Interv Radiol. 2011 Mar;17(1):18-29. [CrossRef] [PubMed]

9. Hansell DM, Bankier AA, MacMahon H, McLoud TC, Müller NL, Remy J. Fleischner Society: glossary of terms for thoracic imaging. Radiology. 2008 Mar;246(3):697722. [CrossRef] [PubMed] 